\title{
A STUDY ON 6T SRAM AND 7T SRAM CELLS
}

\author{
JenniferEunice.R ${ }^{1}$, S. AlimaAashika ${ }^{2}$, T. Bharathisankari ${ }^{3}$ \\ ${ }^{I}$ Professor, ECE, Dr. Sivanthi Aditanar College of Engineering, Tamilnadu, India \\ ${ }^{2}$ Student, ECE, Dr. Sivanthi Aditanar College of Engineering, Tamilnadu, India \\ ${ }^{3}$ Student, ECE, Dr. Sivanthi Aditanar College of Engineering, Tamilnadu, India
}

\begin{abstract}
Nowadays data storage is gaining more importance in human life. All electronic and digital devices need memory for reducing the power consumption. The concept of "more data in less space" is useful for increasing the system performance and overall system efficiency. Generally we used semiconductor memory as "SRAM". SRAM can be abbreviated "Static Random Access Memory". Many VLSI chip can have SRAM memory because of their large storage capacity and fast accessing time. Where, the word static indicates that it does not need to be habitually refreshed but the DRAM need habitually refreshed. DRAM can be abbreviated as "Dynamic Random Access Memory" which is another type of memory. Both the memories can be classified from "Random Access Memory: (RAM). In this paper the power analysis of 6 transistor SRAM is compared with 7 transistor SRAM. As a result the power dissipation of 7 transistors is high when compared with 6 transistors. The power dissipation of 6T SRAM is about $2.991 \mathrm{~mW}$ and the power dissipation of $7 T$ SRAM is about 3.183Mw.SRAM are mostly used for mobile applications, because of their ease of use and low leakage of power. In this paper the schematic of 6T SRAM and 7T SRAM are drawn using DSCH software and the layouts are drawn using MICROWIND software.
\end{abstract}

Keywords: 6T SRAM, 7T SRAM, Power Dissipation.

\section{INTRODUCTION}

In recent days, Static Random Access Memory has become the major part in digital world. Because which occupies the largest portion of SOC (system-on-chip). The device need SRAM memory mainly for device dissipate small amount of power. But the dynamic power dissipation causes problems in digital circuits because the dynamic power depends on supply voltage, switching frequency and output voltage swing. Dynamic power dissipation can be minimized by reducing the supply voltage. At the same time low supply voltage leads to performance degradation and also decreases the threshold voltage which in turn increases the sub threshold current hence the static power dissipation increases. This paper discuss about the power dissipation of 6 transistors and 7 transistors SRAM. It also includes the functional view of $6 \mathrm{~T}$ and 7T SRAM cells.

\section{6T STATIC RANDOM ACCESS MEMORY}

A conventional 6T SRAM consists 6 transistors which form two cross coupled inverters. This bit cell can be read and write single bit data. When a bit is stored in memory the 6T SRAM behave like a latch. The cross coupled inverter pattern which causes large area consumption which is a drawback of 6T SRAM when compared to resistive load. Conventional SRAM with 6 transistors is shown in figure 1 and 6T SRAM have three states they are read, write and hold states.

\subsection{Hold State}

When write operation $(\mathrm{WL}=0)$ the accessible transistor $\mathrm{M} 1$ and M2 disconnect the cell from bit lines. The leakage current can be drawn from $\mathrm{v}_{\mathrm{dd}}$

\subsection{Read State}

The accessible transistor M3 and M6 should be ON when pre-charging bit and bit bar line to high.

\subsection{Write state}

When the $\mathrm{WL}=0$, the value to be written to the bit and bit bar line. Hence we write a data value is " 0 ", we take bit value is " 0 " and bit bar value is " 1 ". and the data value is " 1 ", we take bit value should be " 1 " and the bit bar value is "0".

\section{7T STATIC RANDOM ACCESS MEMORY}

As like 6T SRAM, the 7T SRAM circuit also consists of two CMOS cross coupled to each other. In this circuit we additionally connect NMOS transistor to write line. And it also have two pass NMOS transistor connected to the bit and bit bar line. The access transistor N3 and N4 which are correspondingly connected to the write and read line to perform the write and read operation. Before write operation the 7T SRAM cell depends upon feedback connection. These feedback connection and disconnection can be performed by $\mathrm{N} 5$ transistor. 


\subsection{Write Operation}

The write operation can be start by turning off the N5 transistor to this cut off feedback connection. When N3 is on and N4 is off the bit line bar carries complement of input data. The N5 is turned on and WL is turned off for reconnect the feedback connection to store new data. The bit line bar is discharged to " 0 " for storing " 1 " in the cell. And there is no need to discharge bit line for storing " 0 " in the cell.

\subsection{Read Operation}

When performing the read operation both read and word lines are turned to on and also the transistor N5 is kept on.

\section{RESULTS AND DISCUSSION}

Here we analysis and discuss about the 6T and 7T SRAM cell during read and write operation. And the schematic view of SRAM cell is designed and implemented by using DSCH and MICROWIND software.
Table 1: Comparison of power dissipation between 6T and

\begin{tabular}{|l|l|l|}
\hline OPERATION & 6TSRAM & 7T SRAM \\
\hline VDD & 2.991 & 3.183 \\
\hline BL & 3.084 & 0.292 \\
\hline BLB & 0.329 & 3.925 \\
\hline WL & 5.347 & 3.183 \\
\hline VSS & 3.008 & 2.323 \\
\hline N12 & 0.239 & 12.673 \\
\hline N14 & 2.991 & 1.789 \\
\hline N15 & 2.991 & 3.323 \\
\hline N16 & 2.991 & 3.323 \\
\hline
\end{tabular}

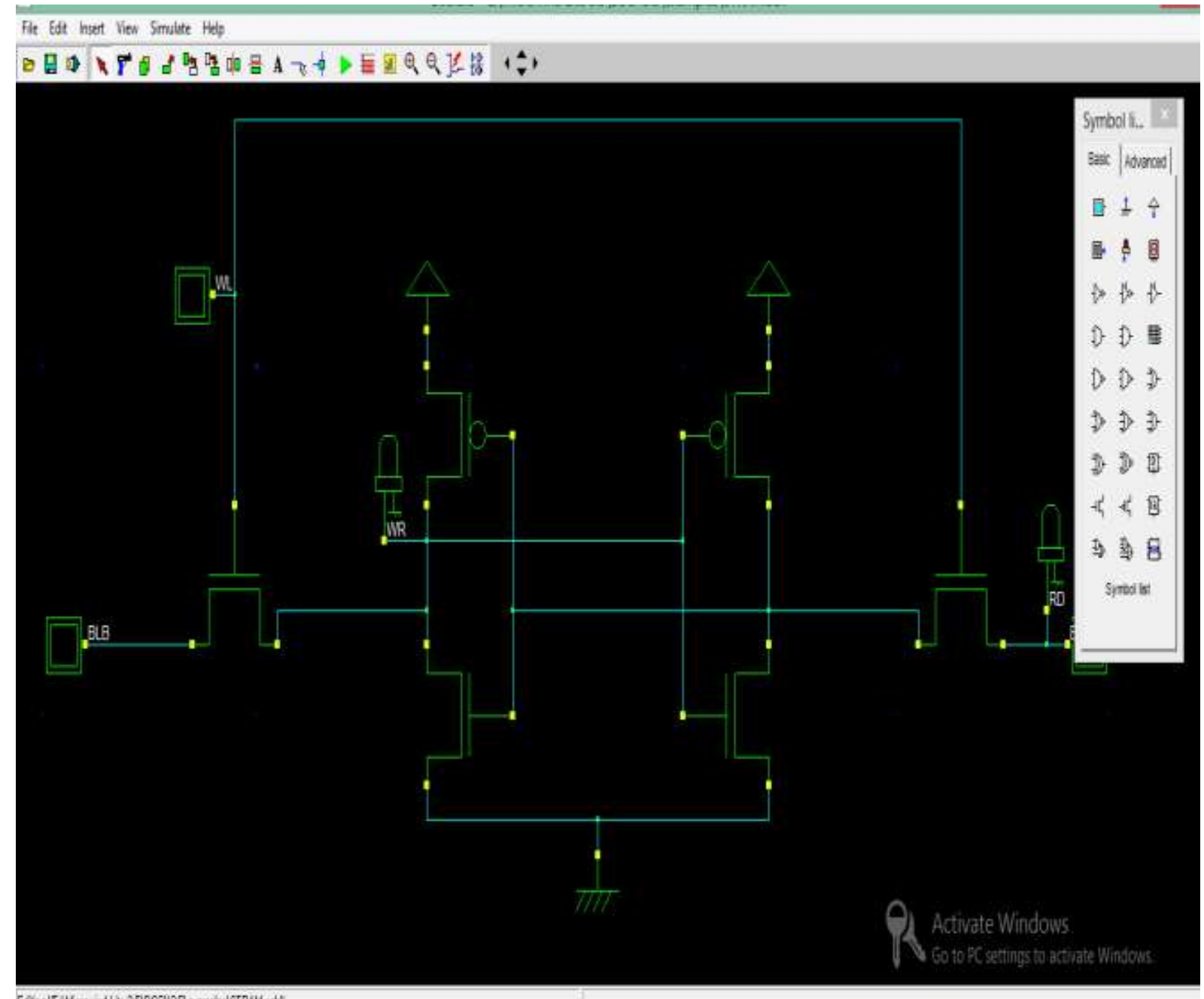

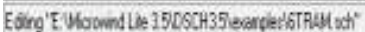

Fig 1: Schematic diagram of 6T SRAM 


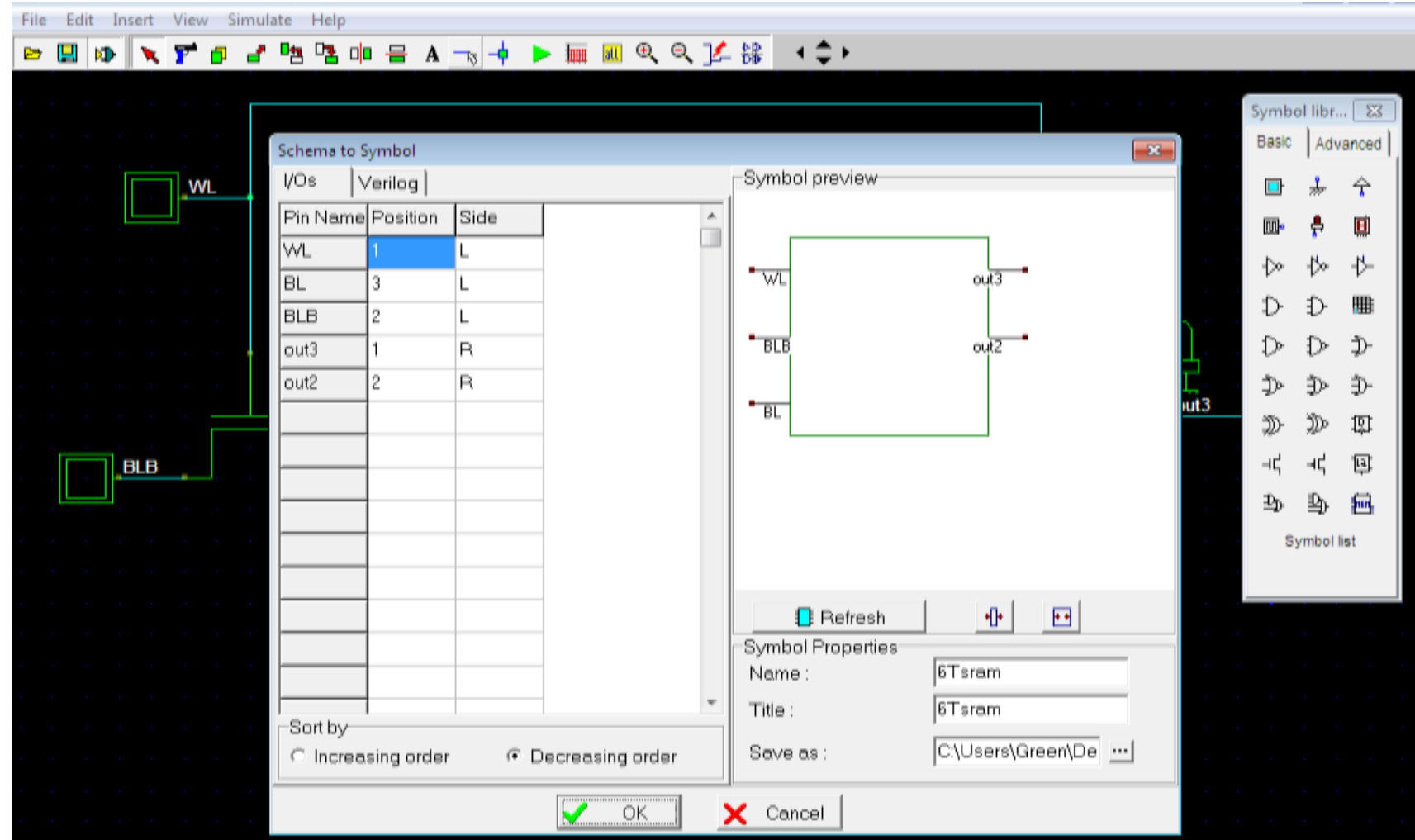

Fig 2: Symbol representation of 6T SRAM

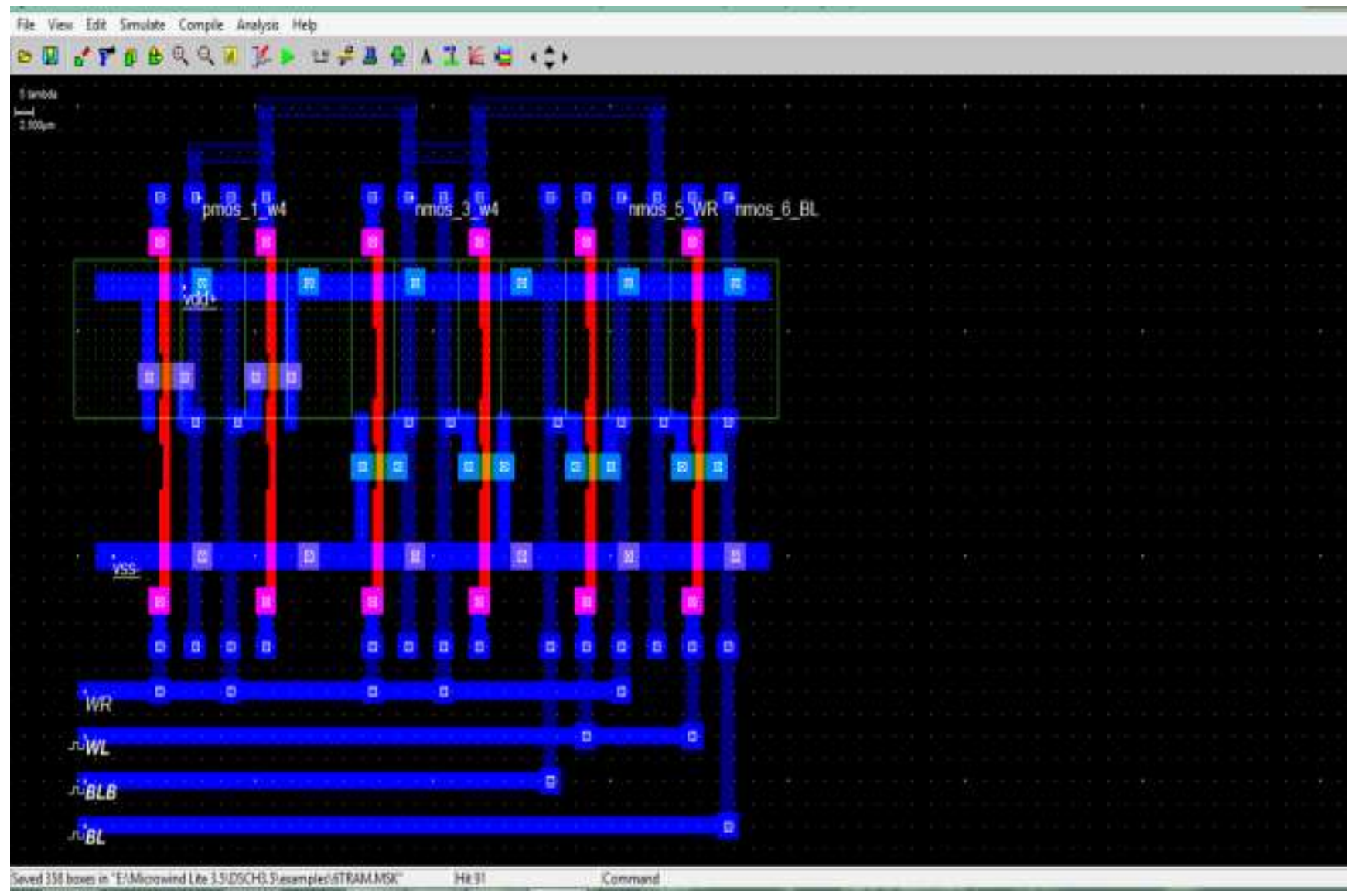

Fig 3: Layout diagram of 6T SRAM 


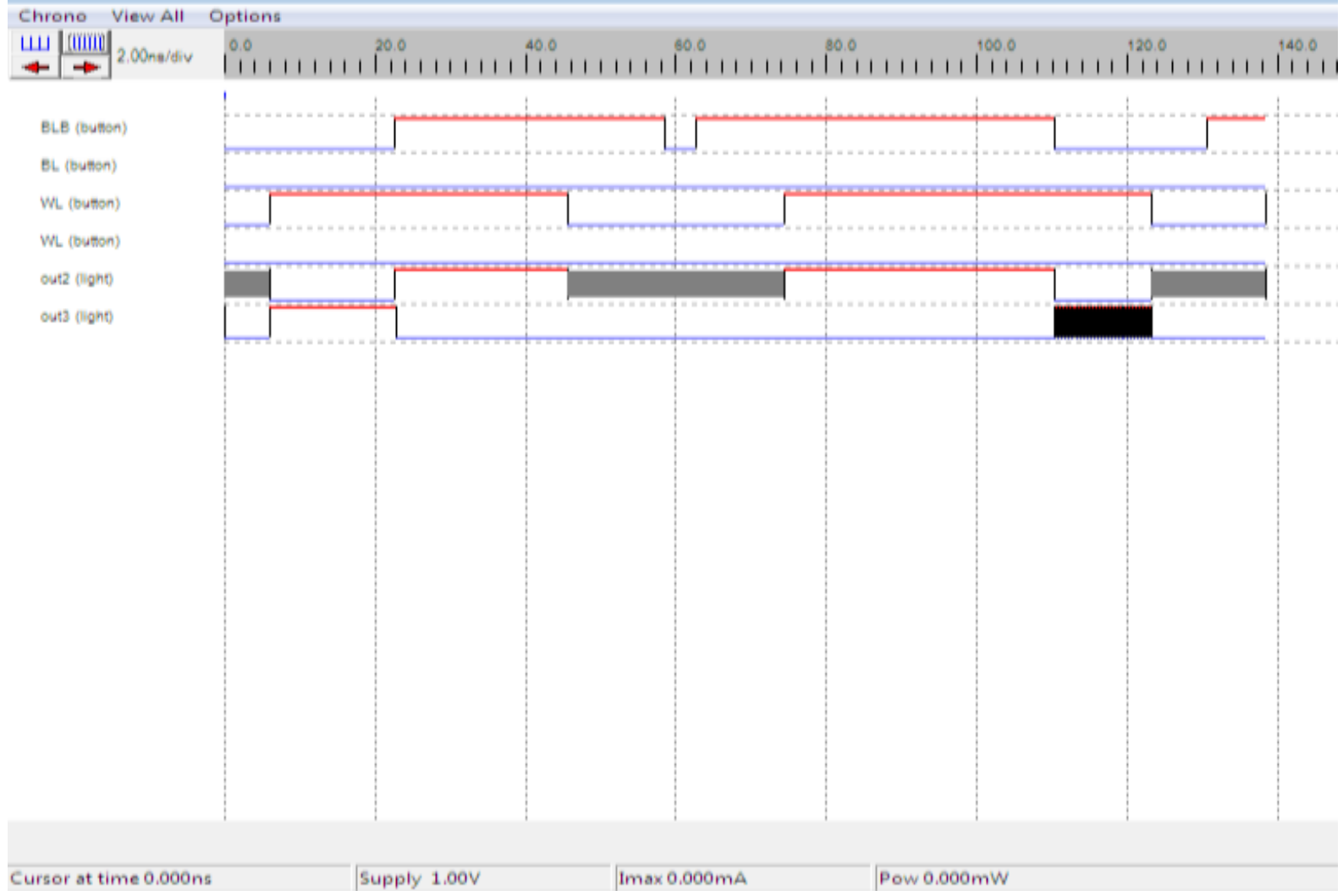

Fig 4: Timing diagram of 6T SRAM

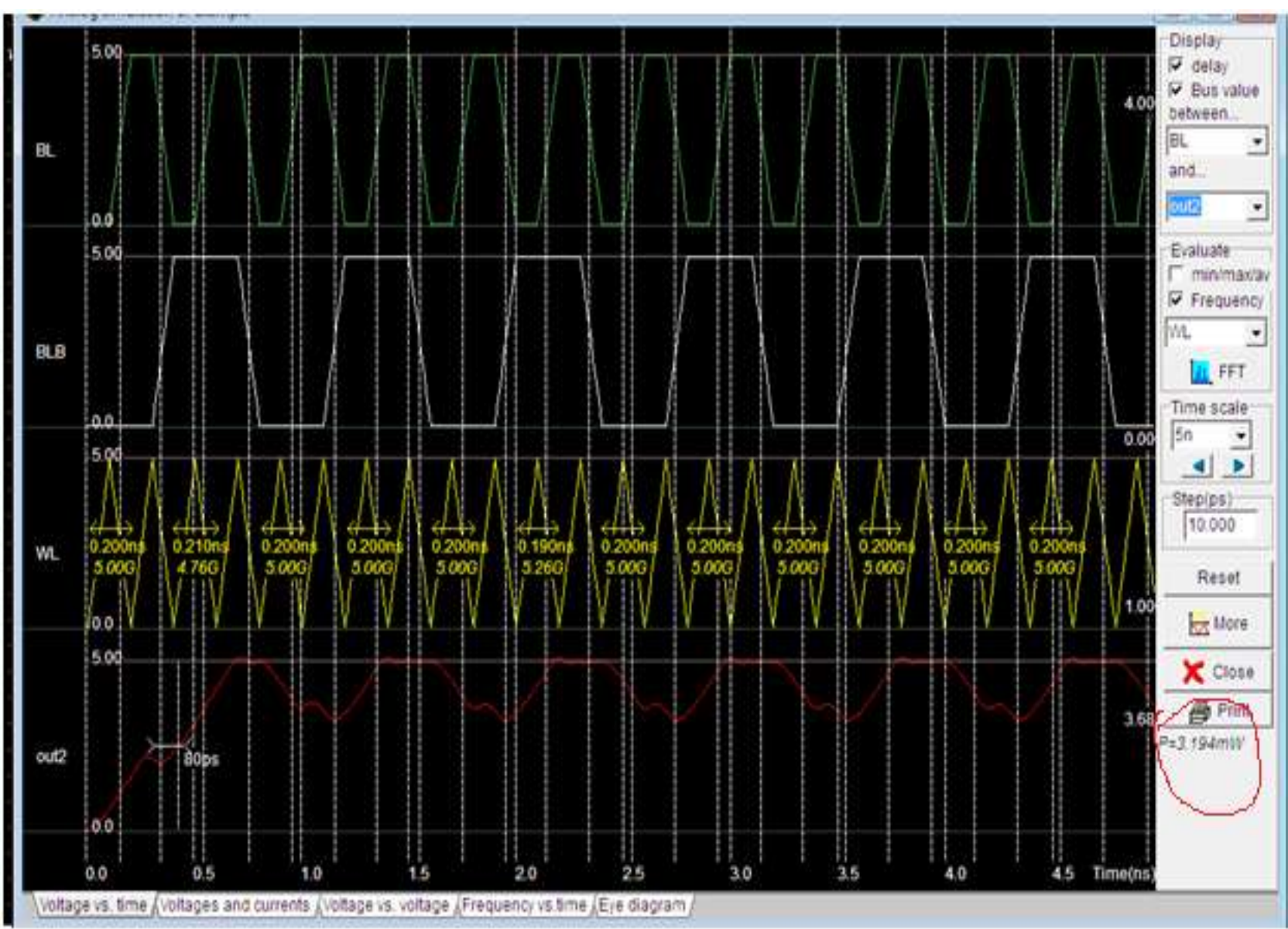

Fig 5: Analog simulation of 6T SRAM 


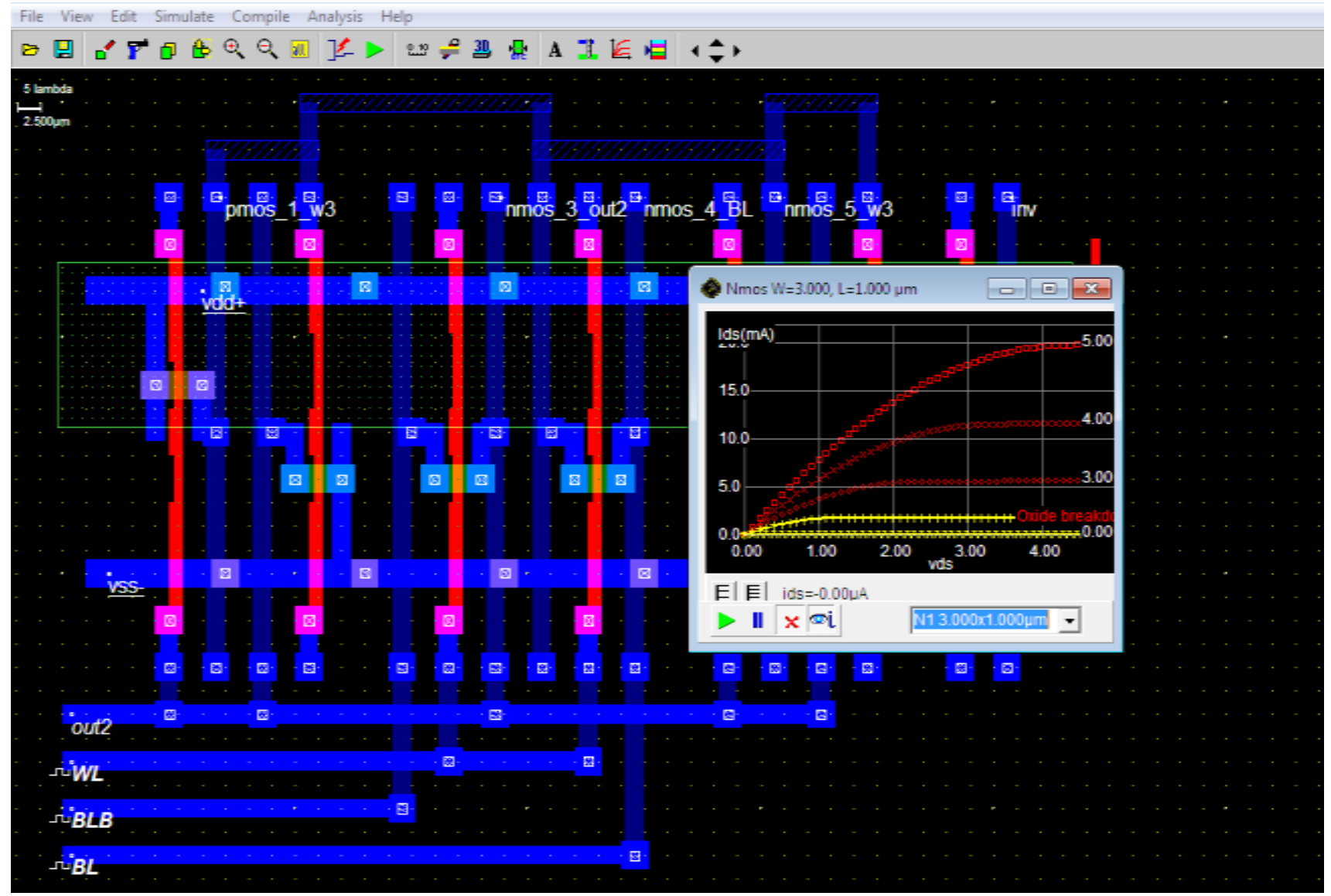

Fig 6: W/L ratio of 6T SRAM

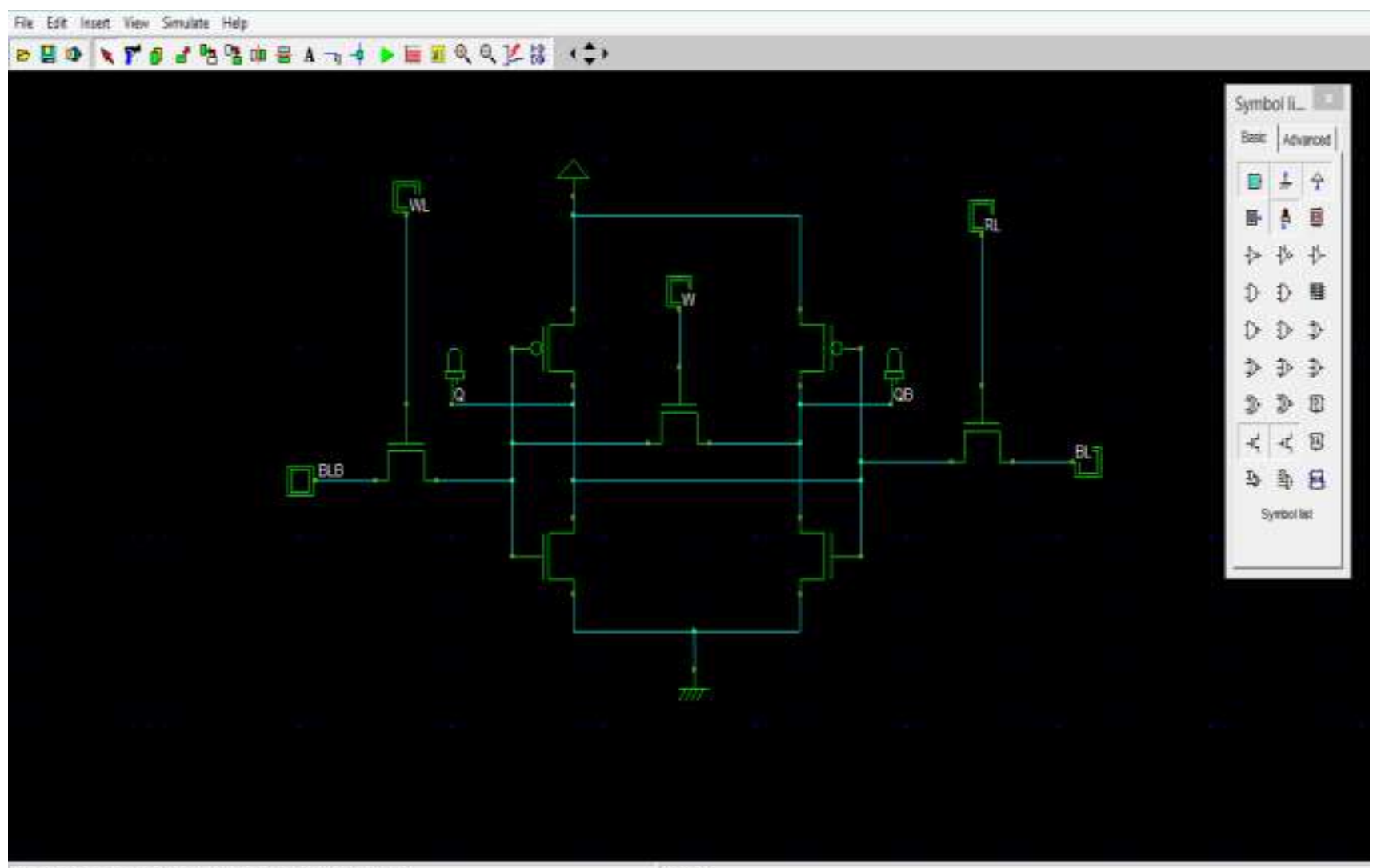

Fig 7: Schematic diagram 7T SRAM 


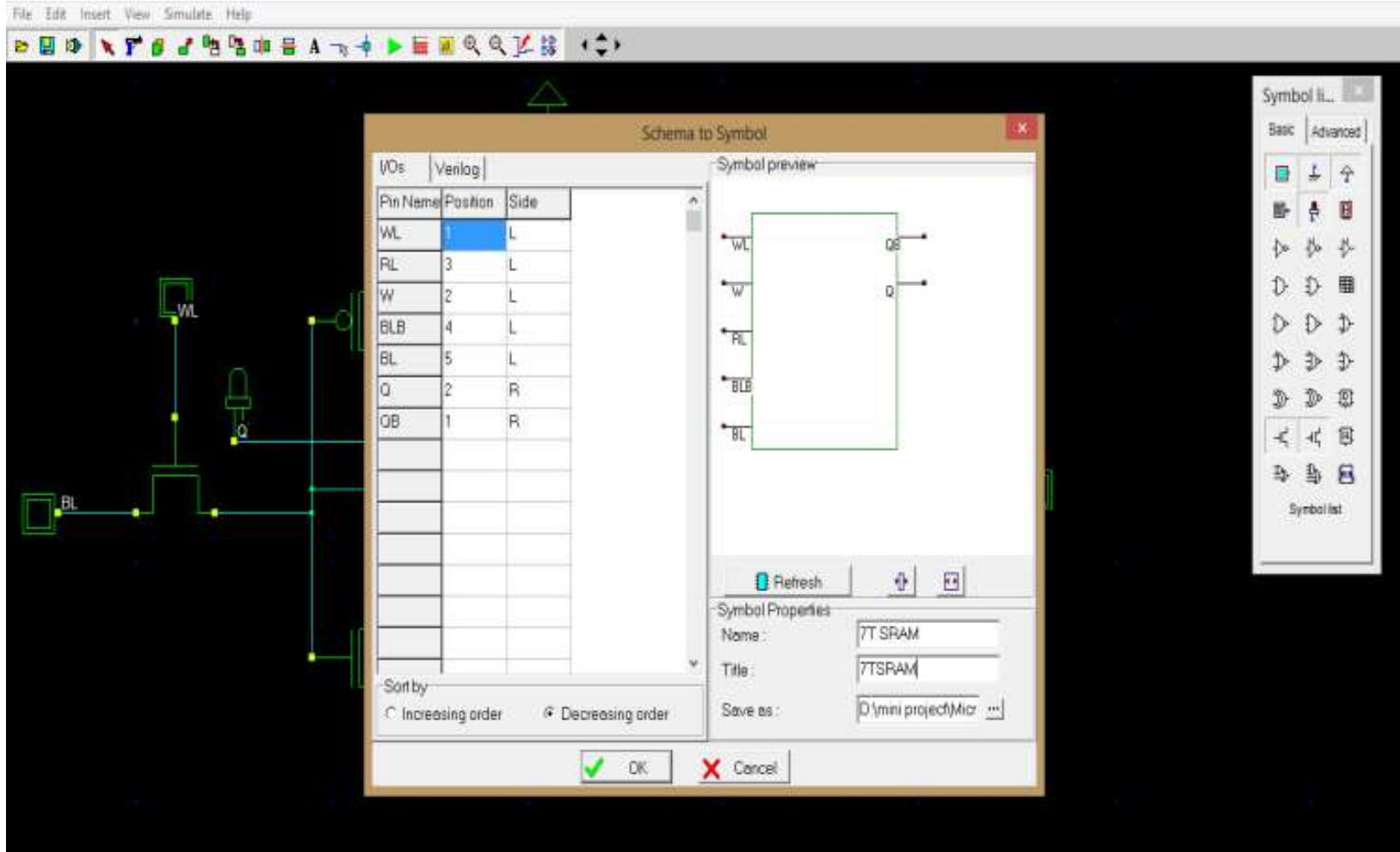

Fig 8: Symbol representation 7T SRAM

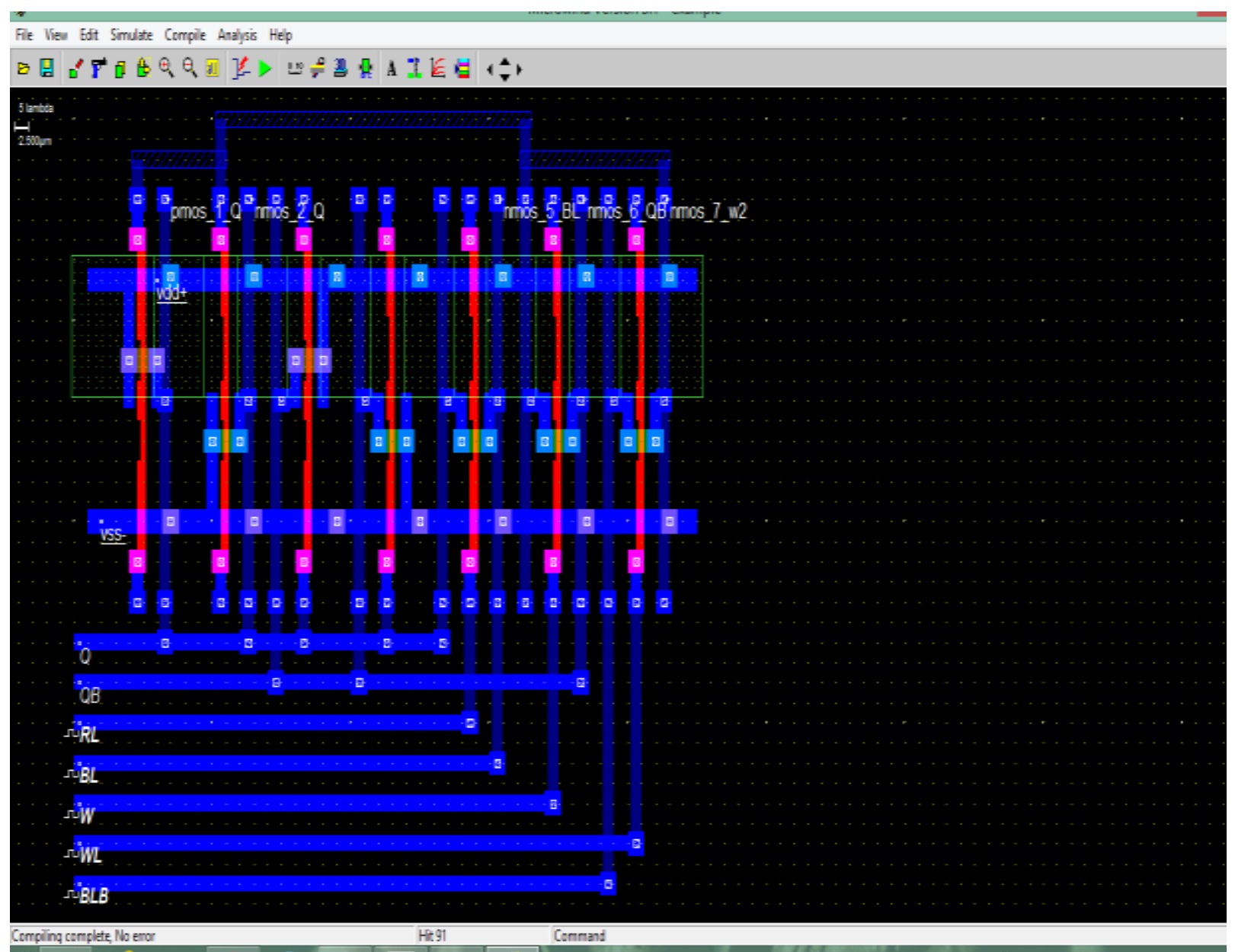

Fig 9: Layout diagram of 7T SRAM 


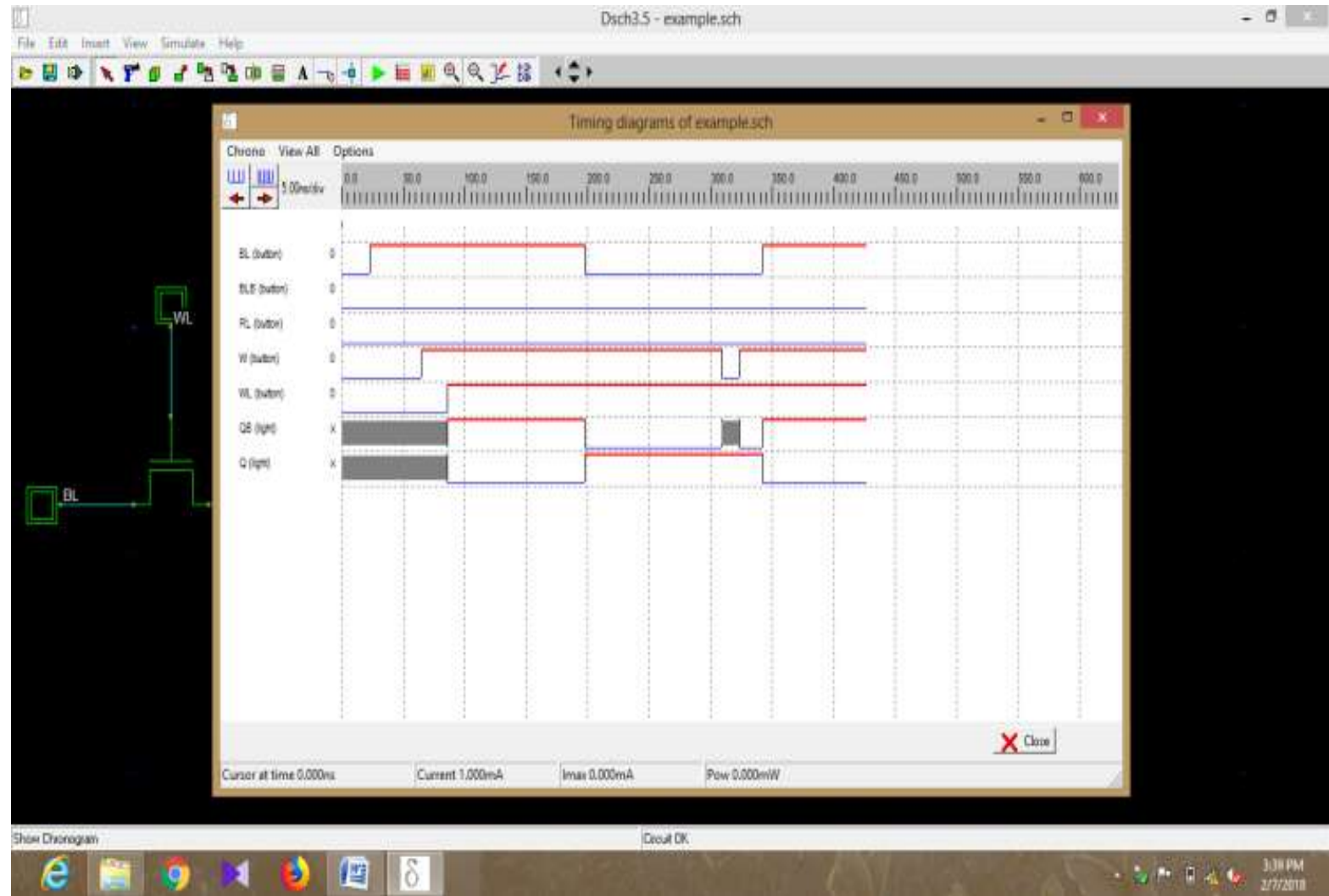

Fig 10: Timing diagram of 7T SRAM

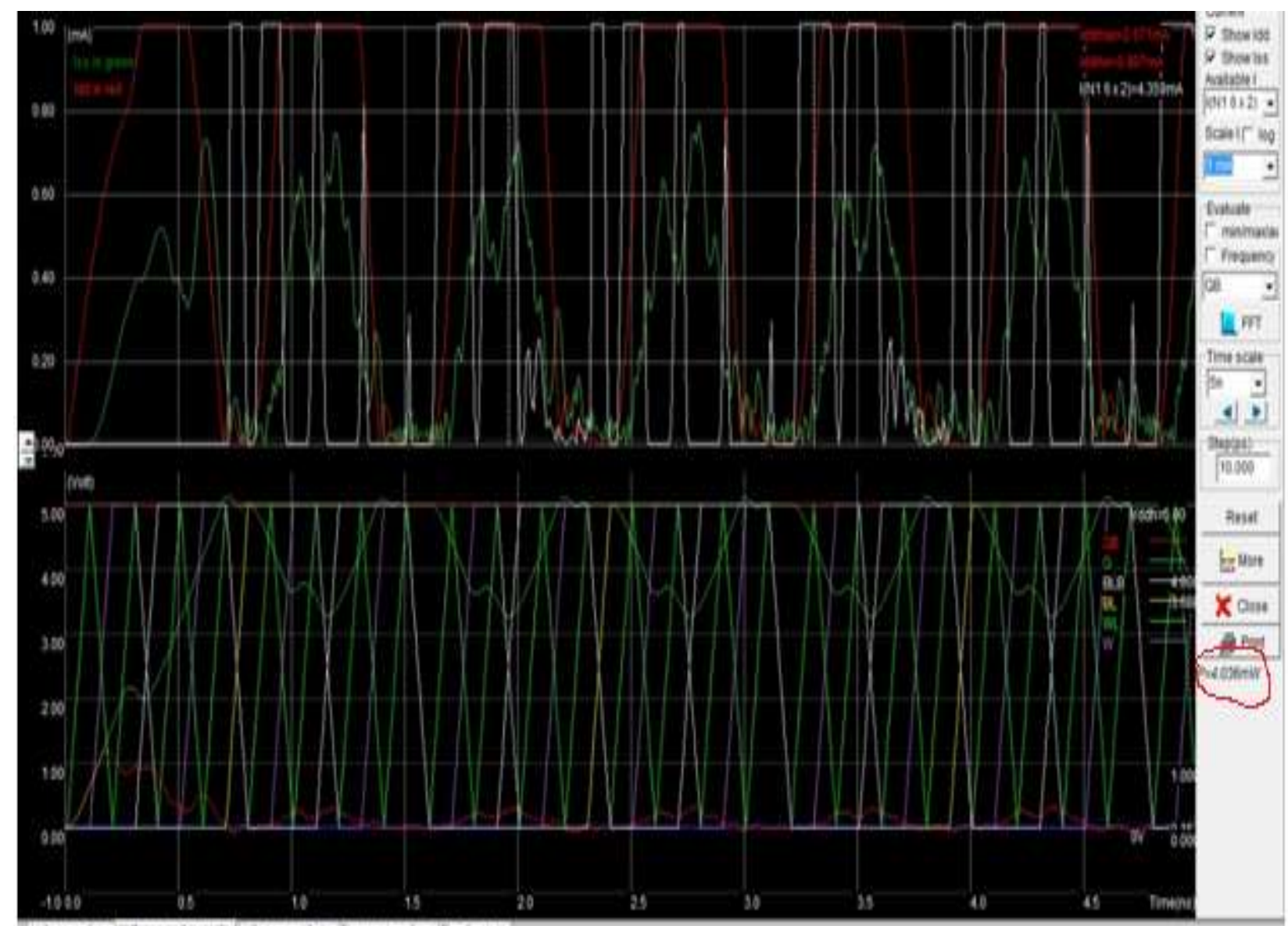

Fig 11: Analog simulation of 7T SRAM 


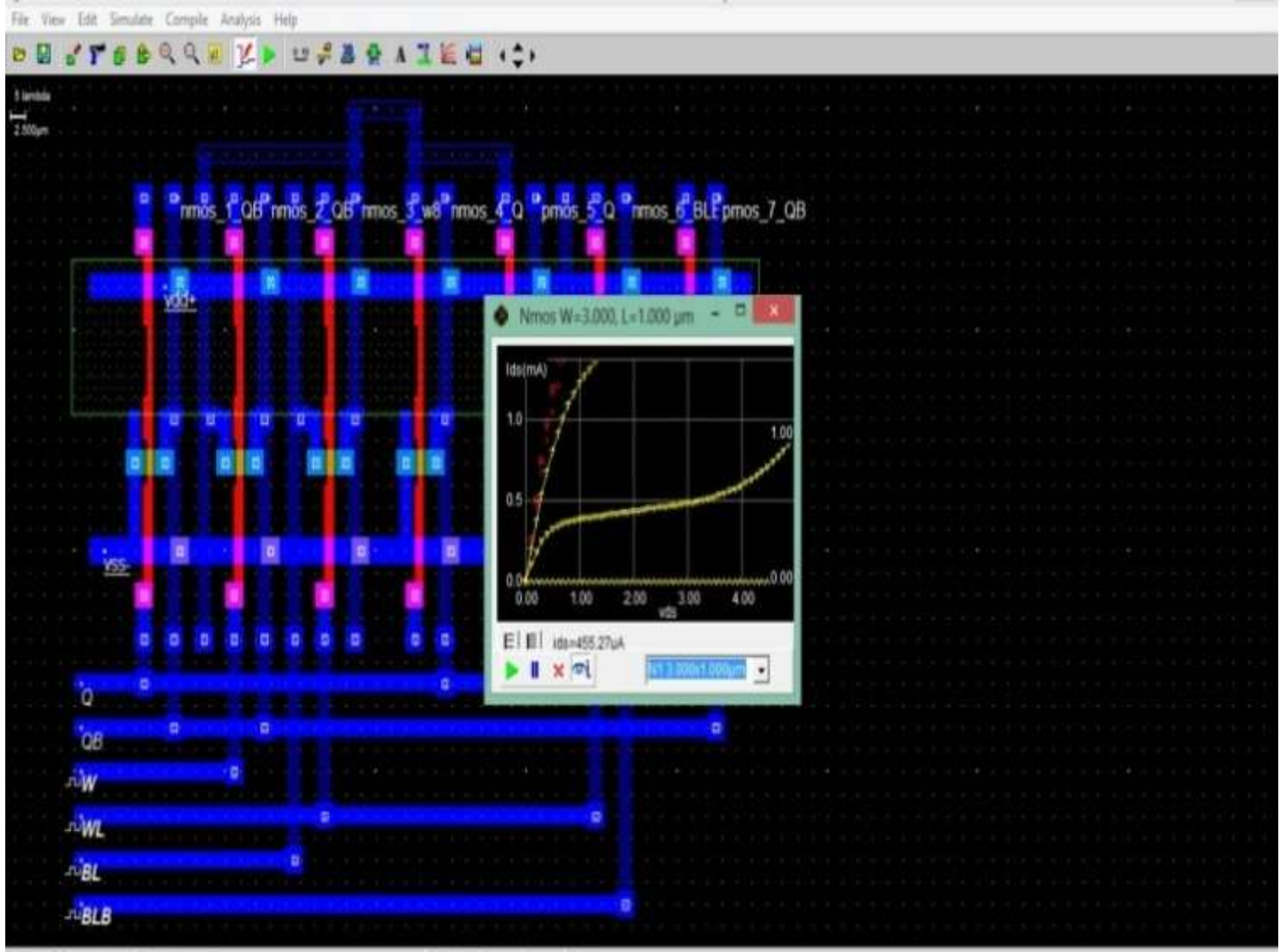

Fig 12: W/L ratio of 7T SRAM

\section{CONCLUSION}

In this paper power dissipation of 6T SRAM is compared with 7T SRAM. Simulation and analysis of 6T SRAM and 7T SRAM is desired. Simulation result shows clearly how read and write operation is performed. It is observed that the power dissipation is less as compared with 6T SRAM to the 7T SRAM in read as well as write mode of operation. In future, power dissipation will play a major role to reduced power consumption.

\section{REFERENCES}

[1]. Islam Aminul, Hassan, Mohd.,"Variability Analysis of 6T and 7T SRAM cell in Sub-45nm Technology," IIUMEngineering Journal, Vol. 12 , No. 1, May 2011, pp. 13-30.

[2]. KaurSimran, and Kumar Ashwani, "Analysis of Low Power SRAM Memory Cell using Tanner Tool," IJECT International Journal Of Electronics \& Communication Technologies, Vol. 3, Issue 1, Jan.-Mar. 2012, pp. 80-84.

[3]. Manoj Kumar, Rohit Kumar "Static Write Margin and Power for 6T \& 7T SRAM Cell: A Comparison" International journal of Advances in Electronics Engineering. pp: 329-333.
[4]. A. P. Chandrakasan and R. W. Broderson, "Minimizing power consumption in digital CMOS circuits," Proc. of the IEEE, vol. 83,no.4,pp. 498- 523, April 1995

[5]. Gu Ming, Yang Jun, Xue Jun, "Low power SRAM design using charge sharing technique," 6th International Conference On ASIC, ASICON,pp.19-23, Oct.2005.

[6]. W. R. E. Aly, and M. A. Bayoumi. (2007). "Low-power cache design using 7T SRAM cell." IEEE Trans. Circ. Sys. Vol. 54, no. 4

\section{BIOGRAPHIES}

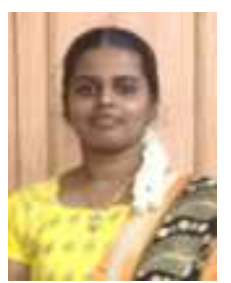

Ms. Jennifer Eunice.R is currently working as Assistant professor in the department of Electronics and Communication at Dr.SivanthiAditanar College of Engineering. Her area of interest includes in the field of VLSI Design and its application in digital electronics, image and signal processing. At present she is guiding students in the field of VLSI design and she is acting as a project co-coordinator for M.E. VLSI Design. 


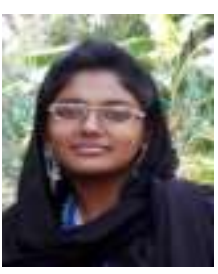

S. AlimaAashika is a final year student in Electronics And Communication Engineering from Dr. Sivanthi Aditnar College of Engineering. Her area of interest includes designing of CMOS in VLSI field. At present she do the project in designing an Antennamodel.

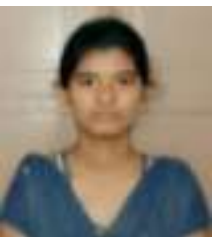

T. Bharathi Sankari is a final year student in Electronics And Communication Engineering from Dr. Sivanthi Aditnar college of Engineering. Her area of interest in circuiting the combinational gates in the field of Digital Electronics. At present she do the project in circuiting prototype modelinantenna related to $R F$ field 